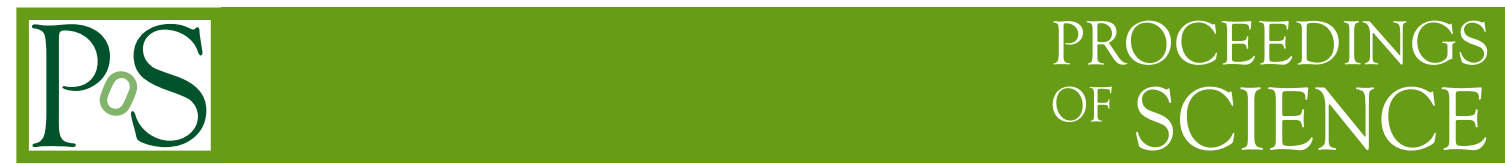

\title{
Recent Heavy Flavor Results from PHENIX
}

\author{
Xiaochun He* for the PHENIX Collaboration \\ Georgia State University \\ E-mail: xheegsu.edu
}

\begin{abstract}
Heavy flavor has played an important role for probing the properties of the hot and dense medium created in relativistic heavy ion collisions both at RHIC and the LHC energy regime. Over the past sixteen years of data taking since year 2000, PHENIX has published a broad spectrum of heavy flavor results which include $J / \psi, \psi^{\prime}$, open heavy flavor, and $\Upsilon$ production in many colliding systems at different collision center-of-mass energies. In this talk, a summary of the most recent heavy flavor results will be given.
\end{abstract}

12th International Workshop on High-pT Physics in the RHIC/LHC Era

2-5 October, 2017

University of Bergen, Bergen, Norway

${ }^{*}$ Speaker. 


\section{Introduction}

After sixteen years of very successful data taking, PHENIX experiment was ended in summer of 2016 in order to make preparation of the next generation experiment, called sPHENIX, at RHIC. PHENIX Collaboration has published extensive results of heavy flavor measurement with a broad rapidity coverage in many colliding systems at variable center of mass energies. This conference proceeding presents the most recent heavy flavor results from PHENIX. The covered topics include $c \bar{c}$ and $b \bar{b}$ cross section measurements in $\mathrm{p}+\mathrm{p}$ and $\mathrm{d}+\mathrm{Au}$ collisions, nuclear modification of heavy flavor production in $\mathrm{Au}+\mathrm{Au}$ collisions, and $\mathrm{B}$ to $J / \psi$ decays in $\mathrm{p}+\mathrm{p}$ and $\mathrm{Cu}+\mathrm{Au}$ collisions. The focus of my talk was to show the flavor separated results from PHENIX which are crucially important for disentangling the response of the hot and dense medium for traversing heavy quarks. A summary and outlook of heavy flavor measurements in the context of sPHENIX experiment is briefly mentioned at the end.

\section{2. $c \bar{c}$ and $b \bar{b}$ Cross Section in $\mathbf{p}+\mathbf{p}$ and $\mathbf{d}+$ Au Collisions}

PHENIX Collaboration has measured $e^{+} e^{-}$pairs from semileptonic heavy flavor decays in central rapidity region $(|y|<0.35)$ in $\mathrm{p}+\mathrm{p}$ and $\mathrm{d}+\mathrm{Au}$ collisions at $\sqrt{s_{N N}}=200 \mathrm{GeV}$ [1]. The $e^{+} e^{-}$ pair yield from $c \bar{c}$ and $b \bar{b}$ is separated by exploiting a double differential fit done simultaneously in the pair mass and $p_{\mathrm{T}}$, as shown in Fig. 1. The data can be well described by PYTHIA as well by MC@NLO and POWHEG event generators.

Based on these simultaneous fits with all three generators to the data, we then extrapolate to $4 \pi$ acceptance and obtained the $c \bar{c}$ and $b \bar{b}$ cross sections, as shown in Fig. 2, both for $\mathrm{p}+\mathrm{p}$ and $\mathrm{d}+\mathrm{Au}$ collisions. The $\mathrm{d}+\mathrm{Au}$ cross section has been scaled down by $N_{\text {coll }}$ to represent the equivalent nucleon-nucleon cross section. We see significant differences for the total cross section in these models. These difference are less pronounced for $b \bar{b}$ than for $c \bar{c}$.

\section{Single Electron Yields from Heavy Flavors in Au+Au Collisions}

PHENIX Collaboration has measured open heavy flavor production in minimum bias $\mathrm{Au}+\mathrm{Au}$ collisions at $\sqrt{s_{N N}}=200 \mathrm{GeV}$ via the yields of electrons from semileptonic decays of heavy flavor hadrons within a rapidity coverage of $|y|<0.35$ [2]. This measurement was made possible, for the first time at RHIC Run11, using the PHENIX silicon vertex detector to measure precision displaced tracking and to extract the relative contributions from charm and bottom hadrons to these electrons as a function of electron $p_{\mathrm{T}}$. We compare the fraction of electrons from bottom hadrons to previously published results extracted from electron-hadron correlations in $\mathrm{p}+\mathrm{p}$ collisions at $\sqrt{s}=200 \mathrm{GeV}$ [3] and find the fractions to be similar within the large uncertainties for $p_{\mathrm{T}}>4$ $\mathrm{GeV} / \mathrm{c}$. We use the bottom electron fractions in $\mathrm{Au}+\mathrm{Au}$ and $\mathrm{p}+\mathrm{p}$ along with the previously measured heavy flavor $R_{A A}$ to calculate the $R_{A A}$ for electrons from charm and bottom decays separately. We find that electrons from bottom hadron decays are less suppressed than those from charm for the region $3<p_{\mathrm{T}}<4 \mathrm{GeV} / \mathrm{c}$, as shown in Fig. 3. During the RHIC Run14, a much larger data set of $\mathrm{Au}+\mathrm{Au}$ collisions at $\sqrt{s_{N N}}=200 \mathrm{GeV}$ was collected by PHENIX. This new data set allowed us to continue exploring the heavy flavor separated electron yield measurement in different event 


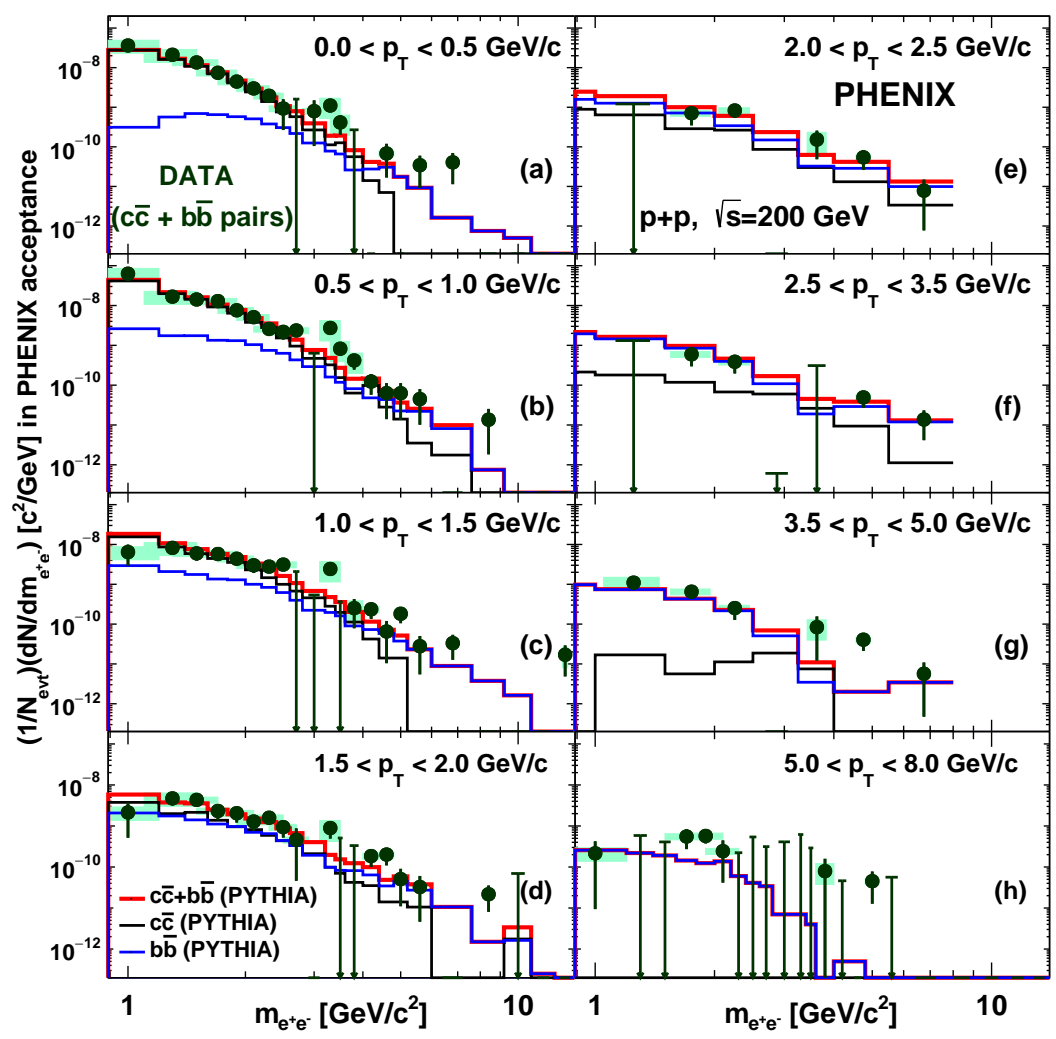

Figure 1: Double differential $e^{+} e^{-}$pair yield from heavy flavor decays in $\mathrm{p}+\mathrm{p}$ collisions at $\sqrt{s}=200 \mathrm{GeV}$ in eight $p_{\mathrm{T}}$ bins. Also shown in the figure are the fitted distributions from PYTHIA event generator. The simulation is fitted to data in the mass region between $1.15<m_{e^{+}} e^{-}<2.4 \mathrm{GeV} / c^{2}$ and $4.1<m_{e^{+}} e^{-}<8.0$ $\mathrm{GeV} / c^{2}$

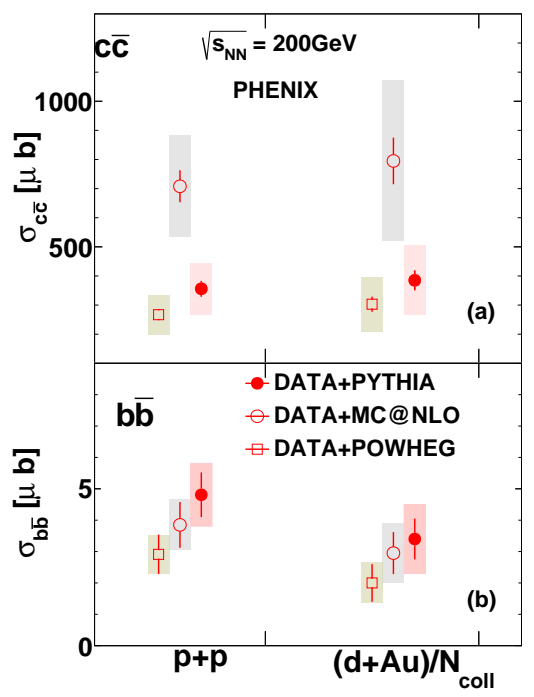

Figure 2: The extracted cross sections of $c \bar{c}$ and $b \bar{b}$ in $\mathrm{p}+\mathrm{p}$ and $\mathrm{d}+\mathrm{Au}$ collision. 
centrality classes. The preliminary results of this ongoing work are shown in Fig. 4. A much stronger suppression of charm production is clearly seen in the most central collisions $(0-10 \%$ centrality bin). Again, within the large uncertainty, the bottom suppression is about the same as seen in the minimum bias events.

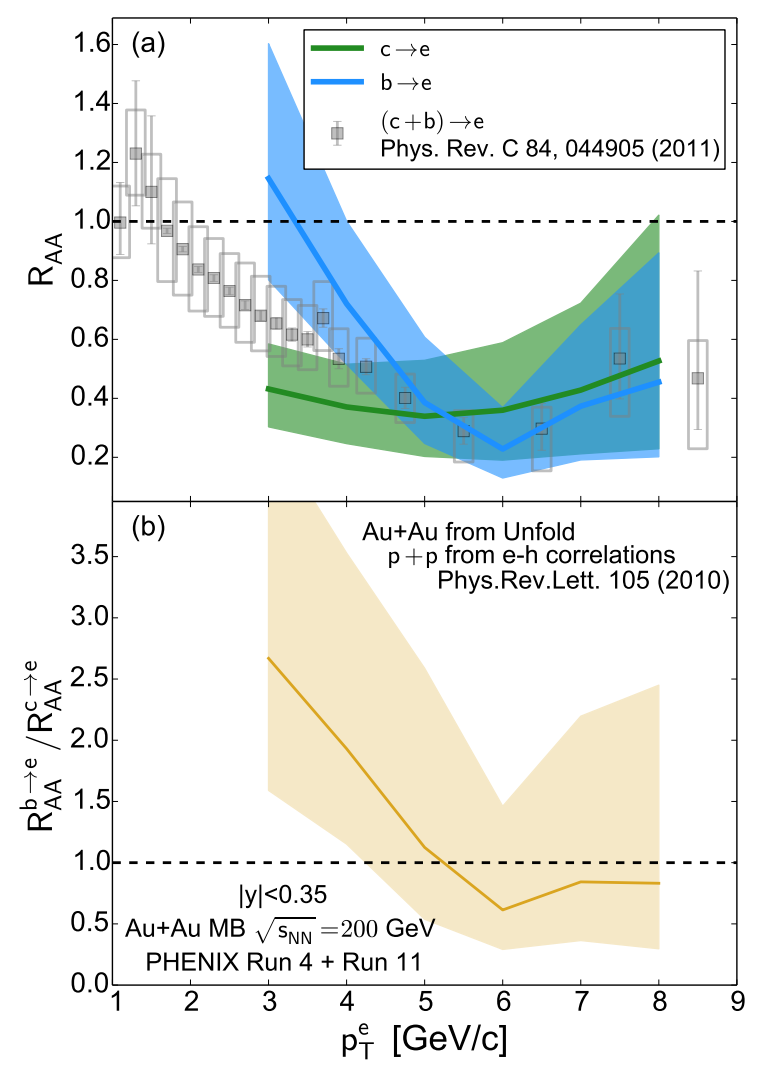

Figure 3: (a) The $R_{A A}$ for $c \rightarrow e, b \rightarrow e$ and combined heavy flavor as a function of electron transverse momentum $\left(p_{T}^{e}\right)$ in minimum bias Au+Au collisions at $\sqrt{s_{N N}}=200 \mathrm{GeV}$ from Run4 and Run11. (b) The ratio of $R_{A A}^{b \rightarrow e} / R_{A A}^{c \rightarrow e}$ as a function of $p_{T}^{e}$.

\section{B to $J / \psi$ Decay Measurements in $\mathbf{p}+\mathbf{p}$ and $\mathrm{Cu}+\mathrm{Au}$ Collisions}

In the forward and backward rapidity regions $(1.2<|y|<2.2)$, PHENIX Collaboration has measured the fraction of $J / \psi$ from B-meson decays in $\mathrm{p}+\mathrm{p}$ and $\mathrm{Cu}+\mathrm{Au}$ collisions at $\sqrt{s_{N N}}=200$ $\mathrm{GeV}$ in RHIC Run12 using the PHENIX forward silicon vertex detector [4]. The extracted fraction is $F_{B \rightarrow J / \psi}=0.025 \pm 0.006$ (stat) \pm 0.010 (syst) for $\mathrm{p}+\mathrm{p}$ collisions (see the left plot in Fig. 5). For $\mathrm{Cu}+\mathrm{Au}$ collisions, $F_{B \rightarrow J / \psi}=0.094 \pm 0.028$ (stat) \pm 0.037 (syst) in the Au-going direction $(-2.2<$ $y<-1.2$ ) and $F_{B \rightarrow J / \psi}=0.089 \pm 0.026$ (stat) \pm 0.040 (syst) in the Cu-going direction $(1.2<y<$ 2.2). The nuclear modification factor, $R_{C u A u}$, of $\mathrm{B}$-mesons in $\mathrm{Cu}+\mathrm{Au}$ collisions is consistent with binary scaling of measured yields in $\mathrm{p}+\mathrm{p}$ collisions at both forward and backward rapidity, as shown in the plot on right in Fig. 5. 

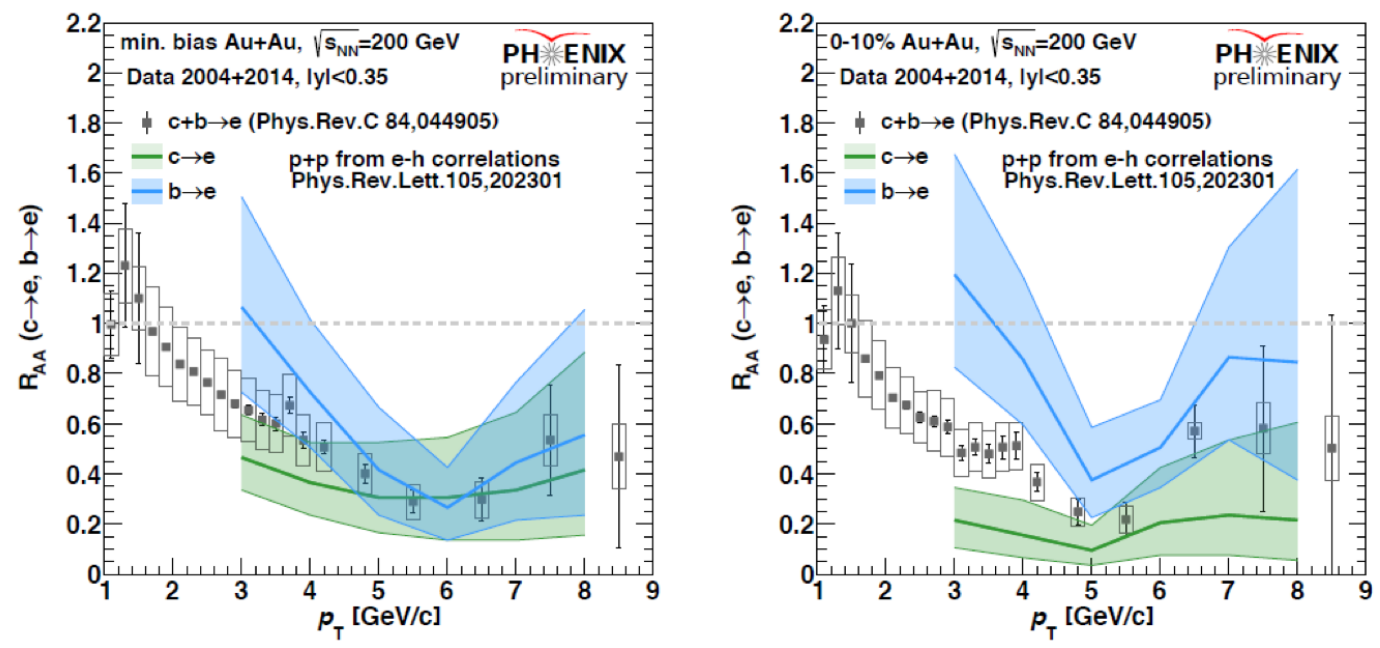

Figure 4: The $R_{A A}$ for $c \rightarrow e, b \rightarrow e$ and combined heavy flavor as a function of electron transverse momentum $\left(p_{T}^{e}\right)$ in minimum bias (left) and in the most central (right) Au+Au collisions at $\sqrt{s_{N N}}=200 \mathrm{GeV}$ from Run4 and Run14 (left).
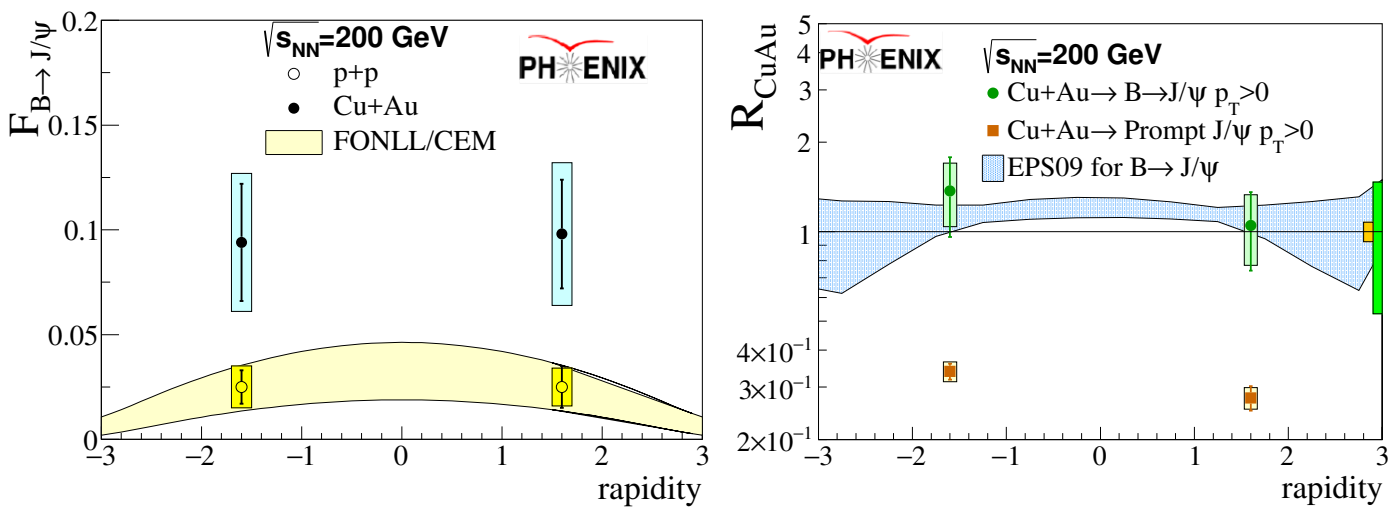

Figure 5: (Left) B-meson to $J / \psi$ decay fraction in $\mathrm{p}+\mathrm{p}$ and $\mathrm{Cu}+\mathrm{Au}$ collisions. The colored band shows a theoretical estimation based on fixed-order plus next-to-leading logs (FONLL) [5] for the B to $J / \psi$ cross section and color evaporation model [6] for the prompt $J / \psi$. (Right) Rapidity dependence of B-meson and prompt $J / \psi$ nuclear modification in $\mathrm{Cu}+\mathrm{Au}$ collisions. The colored band shows the initial state effect estimated from EPS09 [7]. 


\section{Summary and Outlook}

PHENIX measured $c \bar{c}$ and $b \bar{b}$ cross section in $\mathrm{p}+\mathrm{p}$ collisions by simultaneous fit of the dielectron spectra as a function of the pair mass, $p_{\mathrm{T}}$, and $\Delta \phi$ in comparison with three different $\mathrm{pQCD}$ based Monte Carlo models (PYTHIA, MC@NLO and POWHEG). With the silicon vertex detector, PHENIX used an unfolding procedure to infer the parent charm and bottom yields as a function of electron $p_{\mathrm{T}}$ from the semileptonic decays in central rapidity in single electron measurement in $\mathrm{Au}+\mathrm{Au}$ collisions at $\sqrt{s_{N N}}=200 \mathrm{GeV}$. It was found that the nuclear modification (suppression) for electrons from charm is stronger than from bottom hadron decay. We expect a more precision measurement will be available in near future after incorporating the complete Run14 Au+Au data set and better $p+p$ reference in Run 15 . The fraction of B-meson to $J / \psi$ decay is measured in $p+p$ and $\mathrm{Cu}+\mathrm{Au}$ collisions in Run12. The nuclear modification of $\mathrm{B}-$-meson production in $\mathrm{Cu}+\mathrm{Au}$ collision is consistent with binary scaling of measured yields in $p+p$ at both forward and backward rapidity. More heavy flavor results will come from PHENIX with ongoing data analysis. The sPHENIX experiment will provide unprecedented precision measurements of upsilon production and other heavy flavor tagged physics in years to come.

\section{References}

[1] A. Adare et al. (PHENIX Collaboration), Measurements of $e^{+} e^{-}$pairs from open heavy flavor in $p+p$ and $d+A$ collisions at $\sqrt{s_{N N}}=200 \mathrm{GeV}$, Phys. Rev. C 96, 024907 (2017).

[2] A. Adare et al. (PHENIX Collaboration), Single electron yields from semileptonic charm and bottom hadron decays in $A u+A u$ collisions at $\sqrt{s_{N N}}=200 \mathrm{GeV}$, Phys. Rev. C 93, 034904 (2016).

[3] A. Adare et al. (PHENIX Collaboration), Measurement of Bottom Versus Charm as a Function of Transverse Momentum with Electron-Hadron Correlations in $p+p$ Collisions at $\sqrt{s}=200 \mathrm{GeV}$, Phys. Rev. Lett. 103, 082002 (2009).

[4] C. Aidala et al. (PHENIX Collaboration), B-meson production at forward and backward rapidity in $p$ $+p$ and $\mathrm{Cu}+$ Au collisions at $\sqrt{s_{N N}}=200 \mathrm{GeV}$, Phys. Rev. C 96, 064901 (2017).

[5] M. Cacciari, et al., The $p_{T}$ spectrum in heavy-flavour hadroproduction, J. High Energy Phys. 05, 007 (1998); M. Cacciari, et al., QCD Predictions for Charm and Bottom Quark Production at RHIC, Phys. Rev. Lett. 95, 122001 (2005).

[6] A.D. Frawley, T. Ullrich, and R. Vogt, Heavy flavor in heavy-ion collisions at RHIC and RHIC II, Phys. Rep. 462, 125 (2008).

[7] K.J. Eskola, H. Paukkunen, and C.A. Salgado, EPSO9 - A new generation of NLO and LO nuclear parton distribution functions, J. High Energy Phys. 04, 065 (2009). 